\title{
Immune response, clinical outcome and safety of dendritic cell vaccine in combination with cytokine-induced killer cell therapy in cancer patients
}

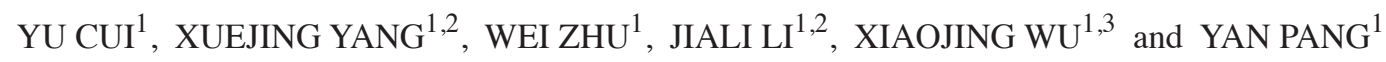 \\ ${ }^{1}$ Department of Oncology, Tianjin Union Medicine Centre, Tianjin 300121; ${ }^{2}$ Shanghai Claison Biotechnology Co., Ltd., \\ Shanghai 201201; ${ }^{3}$ Institute of Oncology, Tianjin Union Medicine Centre, Tianjin 300121, P.R. China
}

Received December 18, 2012; Accepted May 3, 2013

DOI: $10.3892 / 01.2013 .1376$

\begin{abstract}
The aim of the present study was to determine the clinical value of autologous immunocyte therapy as a standard treatment regimen for patients with cancer. A total of 121 patients with cancer were included in this study. Subsequent to performing leukapheresis using the Fresenius Kabi System, $1 \times 10^{7}$ dendritic cells (DCs) for the vaccine and $1 \times 10^{9}$ cytokine-induced killer (CIK) cells for injection were prepared. An analysis of the immune phenotypes of HLA2DR, CD80 and CD83 for the DCs and of CD3, CD8 and CD56 for the CIK cells, as well as negative detection of bacteria and endotoxin, were used as the quality standards. The delayed-type hypersensitivity (DTH) skin test was used to measure the immune response, while physical strength, appetite and sleeping status were analyzed for the clinical outcome. Fever, insomnia, anorexia, joint soreness and skin rashes were recorded as side-effects. Patients received the DC vaccination once a week for six weeks and a CIK cell injection six times within four days. In total, 121 cancer patients with primary tumors located in the colorectum (43.0\%), lung (15.7\%), breast (11.6\%), kidney $(5.8 \%)$, stomach $(4.1 \%)$ and other regions $(19.8 \%)$ were included in the study. A positive cell-mediated cytotoxicity response rate of $76.9 \%$ was detected by the DTH skin tests. Improvements in physical strength, appetite and sleeping status were observed in $94.1,83.9$ and $76.3 \%$ of cases, respectively. None of the serious adverse side-effects that commonly occur during chemotherapy and radiotherapy were observed. During therapy, 69 cases developed a fever that was resolved with antipyretics, dexamethasone or physical cooling, while 28 cases developed insomnia combined with excitement, 19 cases complained of anorexia, 11 cases complained of joint soreness, which was alleviated using analgesics, and 8 cases developed skin rashes. The combined use of CIK cells with a
\end{abstract}

Correspondence to: Professor Yan Pang, Department of Oncology, Tianjin Union Medicine Centre, No. 190 Jieyuan Road, Hongqiao, Tianjin 300121, P.R. China

E-mail: pangyancn@126.com

Key words: dendritic cell vaccine, cytokine-induced killer, cancer
DC-based cancer vaccination strategy may be used to target innate and adaptive immune mechanisms and synergistically promote positive clinical outcomes. The therapy was safe and no serious adverse side-effects similar to those caused by chemotherapy and radiotherapy were observed. The regimen may have a beneficial effect in the future treatment of patients with cancer.

\section{Introduction}

Cancer represents one of the major causes of mortality worldwide (1). More than half of patients suffering from cancer succumb to their condition (1). At present, the major approaches to treating cancer are surgical resection followed by radiation therapy and chemotherapy. These treatments have resulted in significant benefits to patients with the majority of tumor types, and the clinical outcomes have become more satisfactory (2-4). To further increase this trend of improving treatment outcomes, new treatments are necessary; one option proposed for this is immunotherapy (5). It is recognized that multidisciplinary treatments should be used in cancer treatment. The combination of the traditional methods of surgery, chemotherapy and radiotherapy with immunotherapy is a new way for anti-cancer therapies to reduce the mortality of cancer patients. Cancer immunotherapy is a promising cancer treatment method. The dysfunction of the antigen-specific $\mathrm{T}$ cells required to kill the cancer leads to cancer cells being able to grow in cancer patients. Active and adoptive T cell immunotherapies generate $\mathrm{T}$ cells that may be able to target cancer cells $(6,7)$.

Dendritic cells (DCs) are immune cells that function as antigen-presenting cells. They are able to activate naive $\mathrm{CD} 4^{+}$ $\mathrm{T}$ helper cells and unprimed $\mathrm{CD}^{+}$cytotoxic $\mathrm{T}$ lymphocytes. Active immunotherapy, represented by DC-based regimens, has been used to produce tumor-specific antigen-presenting cells and to generate cytotoxic $\mathrm{T}$ lymphocyte responses against cancer cells (8-12). Adoptive immunotherapy, a personalized therapy that uses a patient's own anti-tumor immune cells to kill cancer cells, may be used to treat several types of cancer, representing a potential therapeutic approach against cancer. The adoptive immunotherapy approach is one of the most effective methods for using the body's immune system to treat 
cancer (13). Cytokine-induced killer (CIK) cells are considered to be a heterogeneous population containing the $\mathrm{T}$ cell marker CD3 and the natural killer (NK) cell marker CD56. As an adoptive $\mathrm{T}$ cell immunotherapy, CIK cells have shown significant cytotoxic activity in clinical studies (14-16).

The purpose of the present study was to determine the cellular immune response in terms of the delayed-type hypersensitivity (DTH) skin test and evaluate the subjective clinical outcome and safety of the regimen in cancer patients receiving a DC vaccine in combination with CIK therapy.

\section{Patients and methods}

Study design. The study was an open-label, single-institution, non-randomized exploratory study performed at the Department of Oncology, Tianjin Union Medicine Center, Tianjin, China. All cases were referred to the Department in August 2012. The study protocol was approved by the hospital's Ethics Committees and was in accordance with the 'Treatment with Autologous Immune Cells (T cells and NK cells)' class III medical techniques policy of the Ministry of Health of China. All patients provided written informed consent prior to treatment.

Study procedures. Patients with histologically proven cancer or those who had been diagnosed by imaging and serum tumor markers with histological types that could be defined were selected to participate in the present study. Patients were excluded if they had severe renal or coagulation dysfunction or if the total number of peripheral lymphocytes and monocytes was $<1 \times 10^{9} / 1$. Subsequent to performing leukapheresis using a Fresenius Kabi System with an ECG monitoring system, $1 \times 10^{7} \mathrm{DCs}$ for the vaccine and $1 \times 10^{9} \mathrm{CIK}$ cells for injection were prepared. An analysis of the immune phenotypes of HLA2DR, CD80 and CD83 for the DCs and of CD3, CD8 and CD56 for the CIK cells, as well as negative detection of bacteria and endotoxin were used as the quality standards of the regimen. The DTH skin test was used to measure the immune response, while physical strength, appetite and sleeping status were analyzed for the subjective clinical outcome. Fever, insomnia, anorexia, joint soreness and skin rashes were recorded as side-effects. Patients received DC vaccination once a week for six weeks and CIK therapy six times within four days.

Therapy design. The leukocyte fractions were collected on day 0 . The DCs were reinfused intravenously on days 8, 15 and 22 and intradermally in the bilateral subaxillary or inguinal region by 24-point injection on days 29, 36 and 43. CIK cells were reinfused intravenously once on days 11 and 13 and twice on days 12 and 14 (Table I).

Preparation of DCs and CIK cells (17-20). The patient tumor tissue specimens from the hospital's tissue bank, or human cell lines corresponding to each cancer type, were mechanically dissociated and a single cell suspension was created for each tumor. After disruption by ultrasound and centrifugation at $600 \mathrm{x} \mathrm{g}$ for $30 \mathrm{~min}$, the supernatants were collected as tumor lysate for sensitizing DCs and the DTH test. Patients then underwent leukapheresis with the Fresenius Kabi System. The leukapheresis product, which was enriched for monocytes,
Table I. Regimen of the DC vaccine in combination with CIK therapy in patients with cancer.

\begin{tabular}{|c|c|}
\hline Time & Therapy \\
\hline Day 0 & Collection of leukocyte fractions \\
\hline Day 8 & Reinfusion of DC1 iv \\
\hline Day 11 & Reinfusion of CIK1 iv \\
\hline Day 12 & Reinfusion of CIK 2 iv + CIK 3 iv \\
\hline Day 13 & Reinfusion of CIK4 iv \\
\hline Day 14 & Reinfusion of CIK4 iv + CIK5 iv \\
\hline Day 15 & Reinfusion of DC2 iv \\
\hline Day 22 & Reinfusion of DC3 iv \\
\hline Day 29 & Reinfusion of DC4 id \\
\hline Day 36 & Reinfusion of DC5 id \\
\hline Day 43 & Reinfusion of DC6 id \\
\hline
\end{tabular}

DC, dendritic cell; CIK, cytokine-induced killer.

Table II. DTH skin test following the use of the DC vaccine in combination with CIK therapy.

\begin{tabular}{lcc}
\hline Results of DTH & Definition $(\mathrm{mm})$ & No. $(\%)$ \\
\hline Markedly positive & $>10$ & $32(29.6)$ \\
Positive & $5-10$ & $27(25.0)$ \\
Weakly positive & $2-5$ & $24(22.2)$ \\
Negative & $<2$ & $25(23.1)$ \\
\hline
\end{tabular}

The total number of patients was 108 (data loss in 13 out of 121 cases). DTH, delayed-type hypersensitivity; DC, dendritic cell; CIK, cytokine-induced killer.

was isolated and cultured. Non-adherent cells were cultured in the presence of IFN $\gamma$, CD3 monoclonal antibody, IL-2, IL-1 and autologous plasma for 10 days to form CIK cells. Adherent cells were subsequently cultured for seven days with GM-CSF, IL-4, tumor lysate and TNF to form the DC vaccine.

Quality control of DC vaccine and CIK cells. The prepared DCs and CIK cells were washed. The immune phenotypes of HLA2DR, CD8 and CD83 for the DCs and of CD3, CD8 and CD56 for the CIK cells were analyzed by flow cytometry (FCM). Samples of DCs and CIK cells were cultured for the detection of bacteria, fungus and endotoxin levels. DCs $\left(1 \times 10^{7}\right.$ cells) were resuspended in $4 \mathrm{ml}$ normal saline (NS) in two syringes for intradermal injection and in $100 \mathrm{ml} \mathrm{NS}$ for intravenous injection. The remainder were frozen in $90 \%$ autologous serum and $10 \%$ dimethyl sulfoxide (DMSO) at $1 \times 10^{7} \mathrm{DCs} / \mathrm{ml}$ for further use. The CIK cells $\left(1 \times 10^{9}\right)$ were resuspended in $100 \mathrm{ml} \mathrm{NS}$ for intravenous injection.

Immune response. The DTH skin test was used as the index of the immune response of the DC vaccine in combination with CIK therapy in the patients with cancer. Tumor lysate $(40 \mu \mathrm{g} / 0.1 \mathrm{ml})$ was administrated intradermally into the 
Table III. Subjective clinical outcomes following the use of the DC vaccine in combination with CIK therapy.

\begin{tabular}{lccc}
\hline & \multicolumn{2}{c}{ Improvement in general condition } \\
\cline { 2 - 4 } Characteristics & Significant (\%) & Slight (\%) & $\begin{array}{c}\text { No change } \\
\text { or worse (\%) }\end{array}$ \\
\hline Physical strength & $74(62.7)$ & $37(31.4)$ & $7(5.9)$ \\
Appetite & $37(31.4)$ & $62(52.5)$ & $19(16.1)$ \\
Sleeping status & $36(30.5)$ & $54(45.8)$ & $28(23.7)$ \\
\hline
\end{tabular}

The total number of patients was 118 (data loss in 3 out of 121 cases). DC, dendritic cell; CIK, cytokine-induced killer.

Table IV. Side-effects following the use of the DC vaccine in combination with CIK therapy.

\begin{tabular}{lc}
\hline Characteristics & No. $(\%)$ \\
\hline Fever & $69(58.5)$ \\
Insomnia & $28(23.7)$ \\
Anorexia & $19(16.1)$ \\
Joint soreness & $11(9.3)$ \\
Skin rash & $8(6.8)$ \\
\hline
\end{tabular}

The total number of patients was 118 (data loss in 3 out of 121 cases). A patient may have had more than one side-effect. DC, dendritic cell; CIK, cytokine-induced killer. DC, dendritic cell; CIK, cytokine-induced killer.

forearm of each patient one week after the end of therapy. The results of the DTH test were defined as markedly positive, $>10 \mathrm{~mm}$ diameter of erythema; positive, 5-10 mm; weakly positive, 2-5 $\mathrm{mm}$; and negative, $<2 \mathrm{~mm}$ after $48 \mathrm{~h}$ (Table II).

Subjective clinical outcome. The improvements in the general condition of the patients, including their physical strength, appetite and sleeping status, were evaluated as the subjective clinical outcomes of the therapy (Table III).

Safety. Side-effects of fever, insomnia, anorexia, joint soreness and skin rashes were recorded during the process of the therapy (Table IV).

Statistical analysis. Statistical analyses were performed using the SAS statistical software package (SAS Insititute Inc., Cary, NC, USA). The associations between variables were compared using Pearson's Chi-square test. $\mathrm{P}<0.05$ was considered to indicate a statistically significant difference.

\section{Results}

Patient characteristics. A total of 121 cancer patients with histological diagnoses (78 cases) or imaging and medical history diagnoses (43 cases) were included in the present study. The mean age was 60.3 (range, 30-87) years. There
Table V. Patient characteristics.

\begin{tabular}{lcr}
\hline Characteristic & Value & $\%$ \\
\hline Age (years) & & \\
Range & $30-87$ & \\
Mean \pm SD (range) & $60.3 \pm 15.9$ & \\
Gender, $\mathrm{n}$ & & \\
Male & 62 & 51.2 \\
Female & 59 & 48.8 \\
Diagnosis method, $\mathrm{n}$ & & \\
Histological diagnosis & 78 & 64.5 \\
Imaging and medical history diagnosis & 43 & 35.5 \\
Tumor type, $\mathrm{n}$ & & \\
Colorectal cancer & 52 & 43.0 \\
Lung cancer & 19 & 15.7 \\
Breast cancer & 14 & 11.6 \\
Renal cancer & 7 & 5.8 \\
Gastric cancer & 5 & 4.1 \\
Other & 24 & 19.8 \\
Tumor loading, $\mathrm{n}$ & & \\
With & & \\
Without & & \\
Adjuvant radiotherapy and/or & & \\
chemotherapy, $\mathrm{n}$ & & \\
With & & \\
Without & & \\
\hline
\end{tabular}

CRC, colorectal cancer.

were 62 male and 59 female patients. The primary tumors were located in the colorectum $(43.0 \%)$, lung $(15.7 \%)$, breast $(11.6 \%)$, kidney $(5.8 \%)$, stomach $(4.1 \%)$ and other regions (19.8\%). Among the 121 cases, 72 (59.5\%) had tumor loading in their body and $71(58.7 \%)$ received concurrent radiotherapy and/or chemotherapy (Table V).

Immune response. To test the cell-mediated cytotoxicity response, the DTH skin test was performed at one week subsquent to the end of therapy. Of the 121 patients, 108 underwent the test. Among these 108 cases, 32 were markedly positive, 27 were positive, 24 were weakly positive and 25 were negative $48 \mathrm{~h}$ after the DTH skin-test. In total, a 76.9\% (83/108) immune response rate was achieved by the therapy (Table II).

Subjective clinical outcome. To investigate the subjective clinical outcome, improvements in the physical strength, appetite and sleeping status of the patients were recorded in 118 out of 121 cases. Physical strength was recorded in a total of 111 cases $(94.1 \%)$, with 74 cases of significant and 37 cases of slight improvements in physical strength. Appetite was recorded in a total of 99 cases $(83.9 \%)$, with 37 cases of significant and 62 cases of slight improvement in appetite. Sleeping status was recorded in a total of 90 cases $(76.3 \%)$, with 36 cases of significant and 54 cases of slight improvement in sleeping status (Table III). 
Table VI. Comparison of the results of the DTH test between the DC vaccine and DC vaccine + CIK.

\begin{tabular}{lccccc}
\hline & $\mathrm{DTH}^{+}, \mathrm{n}(\%)$ & $\mathrm{DTH}^{-}, \mathrm{n}(\%)$ & Total, $\mathrm{n}$ & $\chi^{2}$ & P-value \\
\hline "DC vaccine & $4(23.5)$ & $13(76.5)$ & 17 & & $<0.01$ \\
DC vaccine + CIK & $83(76.9)$ & $25(23.1)$ & 108 & 19.74 & \\
Total & 87 & 38 & 125 & & \\
\hline
\end{tabular}

"The data for the DC vaccine therapy alone is cited from our previously reported study (25). DTH, delayed-type hypersensitivity DC, dendritic cell; CIK, cytokine-induced killer.

Safety. The side-effects were recorded in 118 out of 121 cases. None of the serious adverse effects that are common in chemotherapy and radiotherapy were observed. In total, 69 cases developed a fever during the therapy, which was resolved with antipyretics, dexamethasone and/or physical cooling, 28 cases developed insomnia combined with excitement, 19 cases complained of anorexia, 11 cases complained of joint soreness, which was alleviated with analgesics, and 8 cases developed skin rashes (Table IV).

\section{Discussion}

DCs have been widely used for tumor immunotherapy in several types of cancer. The cytotoxic and regulatory antitumor functions of CIK cells have also become attractive targets for immunotherapy. The reciprocal interactions of CIK cells may hold therapeutic promise. The idea of the combined use of CIK cells with DC-based cancer vaccination strategies arises from a number of studies on each strategy $(15,21)$. Targeting the innate and adaptive immune mechanisms may synergistically promote positive clinical outcomes. CIK cells are important in DC-induced antitumor immunity (21-24). DC vaccine regimens in cancer therapy should include evaluations of the CIK cell-stimulating potency. An overview of the effect of the DC vaccine in combination with CIK cell therapy was provided by the present study. In total, $76.9 \%$ of patients were positive for the DTH skin-test at $48 \mathrm{~h}$ post-treatment. The positive rate of the DTH test in cancer patients receiving the DC vaccine in combination with CIK therapy, as reported in the present study, was significantly higher compared with the use of the DC vaccine therapy alone (83/108 vs. 4/17, $\mathrm{P}<0.01$; Table VI), as reported previously (25).

Although decreases in tumor size observed in MRI or CT scans are often considered to be important assessment indices for cancer therapy, it is difficult to evaluate the clinical outcomes of the regimen and the comprehensive results of the whole anti-cancer therapy. For accurate clinical outcomes, the effect of other combined therapies, such as surgery, radiotherapy or chemotherapy, should be excluded. In order to evaluate the clinical outcomes of the regimen, studies should be performed in cancer patients who are restricted to receiving only the regimen of DC vaccination in combination with CIK therapy. However, this situation only occurs in clinical trails and not in routine clinical therapy, in which multidisciplinary therapy is always used to treat patients with cancer. The present study attempted to use the immune response instead of clinical efficiency to demonstrate the therapeutic effect of the regimen of DC vaccination in combination with CIK therapy (26).

It is generally considered that the detection of the cell surface phenotypes of peripheral blood T lymphocytes by FCM prior to and following DC vaccine therapy is a standard method for evaluating the effect of therapy on the immune function of patients. However, the DTH skin test, with the advantages of being simple to perform, cheap in economy and efficient at indicating an immune response, may be an alternative choice of efficiency index for indicating the clinical response of cancer patients undergoing DC vaccination in combination with CIK therapy $(27,28)$. A positive DTH response indicates that the DC vaccine and CIK cells injected into the body have affected the patients' immune system, suggesting that the regimen had an immunological function, which should correspond to clinical anti-cancer efficiency. As well as the increase in the immune responses of patients in the present study, the subjective clinical outcomes improved rapidly. In total, 94.1, 83.9 and $76.3 \%$ of cases exhibited improvements in physical strength, appetite and sleeping status, respectively.

The combined therapy was safe and could be performed on outpatients. No serious adverse side-effects similar to those caused by chemotherapy and radiotherapy were observed in all 121 cases. During the therapy, 58.5\% of cases developed a fever, $23.7 \%$ of cases developed insomnia combined with excitement, $16.1 \%$ of cases complained of anorexia, $9.3 \%$ of cases complained of joint soreness and $6.8 \%$ exhibited skin rashes. All these adverse side-effects were well-tolerated and usually alleviated with the relevant treatments.

Patients with advanced cancer usually have a bad general condition due to the development of cancer and repeated use of surgery, radiotherapy or chemotherapy, which may be harmful to the patients in terms of making the treatment ineffective and increasing the susceptibility to tumor progression. The most promising characteristic of the regimen of DC vaccination in combination with CIK treatment in cancer therapy was its low toxicity. Therefore, the regimen was generally well-tolerated with good compliance.

There were no serious adverse side-effects due to the regimen of DC vaccination in combination with CIK cancer therapy. The DC vaccine and CIK cells injected into the body were able to stimulate the patient's immune systems against the cancer. The regimen may be beneficial to the future treatment of patients with cancer. 


\section{Acknowledgements}

The present study was partially supported by the Tianjin Municipal Science and Technology Commission (No. 12ZCDZSY17100).

\section{References}

1. Jemal A, Siegel R, Ward E, Hao Y, Xu J, Murray T and Thun MJ: Cancer statistics, 2008. CA Cancer J Clin 58: 71-96, 2008.

2. Videtic GM: Locally advanced non-small cell lung cancer: what is the optimal concurrent chemoradiation regimen? Cleve Clin J Med 79 (Electronic Suppl 1): eS32-eS37, 2012.

3. Ishikura S: Optimal radiotherapy for non-small-cell lung cancer: current progress and future challenges. Gen Thorac Cardiovasc Surg 60: 127-131, 2012.

4. Bretthauer M: Evidence for colorectal cancer screening. Best Pract Res Clin Gastroenterol 24: 417-425, 2010.

5. Niccolai E and Amedei A: Vaccine immunotherapy strategies in colorectal cancer treatment. Single Cell Biol 1: e1000102, 2012.

6. Yaddanapudi K, Mitchell RA, Putty K, Willer S, Sharma RK, Yan J, Bodduluri H and Eaton JW: Vaccination with embryonic stem cells protects against lung cancer: is a broad-spectrum prophylactic vaccine against cancer possible? PLoS One 7: e42289, 2012.

7. Armstrong AC and Gilham DE: Targeting breast cancer vaccines to dendritic cells: improved immunological responses with less protein? Breast Cancer Res 14: 106, 2012.

8. Kalinski P and Okada H: Polarized dendritic cells as cancer vaccines: directing effector-type T cells to tumors. Semin Immunol 22: 173-182, 2010.

9. Steinman RM: Decisions about dendritic cells: past, present, and future. Annu Rev Immunol 30: 1-22, 2012.

10. Barth RJ Jr, Fisher DA, Wallace PK, Channon JY, Noelle RJ, Gui J and Ernstoff MS: A randomized trial of ex vivo CD40L activation of a dendritic cell vaccine in colorectal cancer patients: tumor-specific immune responses are associated with improved survival. Clin Cancer Res 16: 5548-5556, 2010.

11. Lesterhuis WJ, de Vries IJ, Schreibelt G, Lambeck AJ, Aarntzen EH, Jacobs JF, Scharenborg NM, van de Rakt MW, de Boer AJ, Croockewit S, van Rossum MM, Mus R, Oyen WJ, Boerman OC, Lucas S, Adema GJ, Punt CJ and Figdor CG: Route of administration modulates the induction of dendritic cell vaccine-induced antigen-specific $\mathrm{T}$ cells in advanced melanoma patients. Clin Cancer Res 17: 5725-5735, 2011.

12. Gilboa E: DC-based cancer vaccines. J Clin Invest 117: 1195-1203, 2007.

13. Ma Y, Zhang Z, Tang L, Xu YC, Xie ZM, Gu XF and Wang HX: Cytokine-induced killer cells in the treatment of patients with solid carcinomas: a systematic review and pooled analysis. Cytotherapy 14: 483-493, 2012.

14. Joshi PS, Liu JQ, Wang Y, Chang X, Richards J, Assarsson E, Shi FD, Ljunggren HG and Bai XF: Cytokine-induced killer $\mathrm{T}$ cells kill immature dendritic cells by TCR-independent and perforin-dependent mechanisms. J Leukoc Biol 80: 1345-1353, 2006.

15. Zhang S, Wang Q, Li WF, Wang HY, Zhang HJ and Zhu JJ: Different antitumor immunity roles of cytokine activated $\mathrm{T}$ lymphocytes from naive murine splenocytes and from dendritic cells-based vaccine primed splenocytes: implications for adoptive immunotherapy. Eksp Onkol 26: 55-62, 2004.
16. Nagaraj S, Ziske C and Schmidt-Wolf IG: Human cytokine-induced killer cells have enhanced in vitro cytolytic activity via non-viral interleukin-2 gene transfer. Genet Vaccines Ther 2: 12, 2004.

17. Frank MO, Kaufman J, Tian S, Suárez-Fariñas M, Parveen S, Blachère NE, Morris MJ, Slovin S, Scher HI, Albert ML and Darnell RB: Harnessing naturally occurring tumor immunity: a clinical vaccine trial in prostate cancer. PLoS One 5: e12367, 2010.

18. Nicol AJ, Tazbirkova A and Nieda M: Comparison of clinical and immunological effects of intravenous and intradermal administration of $\alpha$-galactosylceramide (KRN7000)-pulsed dendritic cells. Clin Cancer Res 17: 5140-5151, 2011.

19. Zhang SN, Choi IK, Huang JH, Yoo JY, Choi KJ and Yun CO: Optimizing DC vaccination by combination with oncolytic adenovirus coexpressing IL-12 and GM-CSF. Mol Ther 19: 1558-1568, 2011.

20. Ma Y, Zhang Z, Tang L, Xu YC, Xie ZM, Gu XF and Wang HX: Cytokine-induced killer cells in the treatment of patients with solid carcinomas: a systematic review and pooled analysis. Cytotherapy 14: 483-493, 2012.

21. Chang XH, Cheng HY, Cheng YX, Ye X, Guo HF, Fu TY, Zhang L, Zhang G and Cui H: Specific immune cell therapy against ovarian cancer in vivo and in vitro. Ai Zheng 27: 1244-1250, 2008 (In Chinese).

22. Kim BR, Yang EK, Kim DY, Kim SH, Moon DC, Lee JH, Kim HJ and Lee JC: Generation of anti-tumour immune response using dendritic cells pulsed with carbonic anhydrase IX-Acinetobacter baumannii outer membrane protein A fusion proteins against renal cell carcinoma. Clin Exp Immunol 167: 73-83, 2012.

23. Schwaab T, Schwarzer A, Wolf B, Crocenzi TS, Seigne JD, Crosby NA, Cole BF, Fisher JL, Uhlenhake JC, Mellinger D, Foster C, Szczepiorkowski ZM, Webber SM, Schned AR, Harris RD, Barth RJ Jr, Heaney JA, Noelle RJ and Ernstoff MS: Clinical and immunologic effects of intranodal autologous tumor lysate-dendritic cell vaccine with Aldesleukin (Interleukin 2) and IFN-(alpha)2a therapy in metastatic renal cell carcinoma patients. Clin Cancer Res 15: 4986-4992, 2009.

24. Jähnisch H, Füssel S, Kiessling A, Wehner R, Zastrow S, Bachmann M, Rieber EP, Wirth MP and Schmitz M: Dendritic cell-based immunotherapy for prostate cancer. Clin Dev Immunol 2010: 517493, 2010.

25. Liu Y, Zhang W, Zhang B, Yin X and Pang Y: DC Vaccine therapy combined concurrently with oral capecitabine in metastatic colorectal cancer patients. Hepatogastroenterology 60: hge12522, 2012.

26. Kalos M: Biomarkers in T cell therapy clinical trials. J Transl Med 9: 138, 2011.

27. Soliman H: Developing an effective breast cancer vaccine. Cancer Control 17: 183-190, 2010.

28. Hoos A, Eggermont AM, Janetzki S, Hodi FS, Ibrahim R, Anderson A, Humphrey R, Blumenstein B, Old L and Wolchok J: Improved endpoints for cancer immunotherapy trials. J Natl Cancer Inst 102: 1388-1397, 2010. 